\title{
Improving Wi-Fi based Indoor Positioning using Particle Filter based on Signal Strength
}

\author{
${ }^{+*}$ Md.Sabbir Rahman Sakib, ${ }^{+}$Md Abdul Quyum, ${ }^{+}$Karl Andersson, ${ }^{+}$Kåre Synnes, ${ }^{*}$ Ulf Körner \\ ${ }^{+}$Department of Computer Science, Electrical \& Space Engineering, Luleå University of Technology, Sweden \\ ${ }^{*}$ Department of Electrical \& Information Technology, Lund University, Sweden \\ sakib3@gmail.com,mdaquy-0@student.ltu.se,karl.andersson@1tu.se, kare.synnes@ltu.se, ulf.korner@eit.lth.se
}

\begin{abstract}
Indoor positioning is recognized as one of the upcoming major applications which can be used in wide variety of applications such as indoor navigation and enterprise asset tracking. The significance of localization in indoor environments have made the use of Wi-Fi based indoor positioning so that it can utilize available current wireless infrastructure and perform positioning very easily. In this paper we introduced a user friendly prototype for Wi-Fi based indoor positioning system where a user can identify its own position in indoor. Wi-Fi received signal strength (RSS) fluctuations over time introduce incorrect positioning. To minimize the fluctuation of RSS, we developed Particle Filters with the prototype. A comparison between with and without Particle Filter for error performance is presented and at the same time it is also noticed that variation in number of particles could change the positioning accuracy. Moreover comparison between calibration data in all directions and in one direction while constructing a radio map is presented.
\end{abstract}

Keywords-positioning; rss fingerprinting; particle filters; radio map; calibration data;

\section{INTRODUCTION}

Today location awareness is becoming very important to all of us. Our daily lifestyle demands multitude of location based applications. Depending on demand there are many applications those have different features. Indoor positioning has location awareness issues in indoor environments e.g. inside a building or shopping mall or hospital or library and so on. When we talk about indoor positioning, we pre-assumed that asset or user is in indoor environments. If not then user or asset is outside. An interesting statistics is found that people spend $80-90 \%$ of their time in indoor and $70 \%$ cellular calls and $80 \%$ data connections are originated from indoor [2]. It motivates us to find a suitable solution for indoor positioning.

\section{Why Wi-Fi BASED INDOOR POSITIONING ?}

Nowadays, the available location aware services are provided by mobile phones, based on GPS. Although GPS has a very high signal level degradation which makes it unsuitable for indoor environments but it is popular for outdoor environments because of excellent performance. Right now every smart phones contain Wireless LAN interface where a user can connect to a network in a regular fashion from available access point(Wi-Fi hotspot). So Wi-Fi based indoor positioning is a technique that can use current available Wireless LAN infrastructure to solve the problem of positioning issues in indoor environment.

\section{CHARACTERISTICS OF WI-FI IN INDOOR ENVIRONMENT}

According to the FCC regulation WLAN should operate at very low power level (1 watt or $30 \mathrm{dBm}$ ). After penetrating into a $25 \mathrm{~cm}$ concrete wall, $16.61 \mathrm{dBm}$ signal attenuates which is sufficient to degrade the positioning accuracy[3]. While holding a mobile phone, a user itself can act as an obstacle between mobile phone and APs. As a result signal may drop $10-15 \mathrm{dBm}$ which is $14-21 \%$ of total effective signal strengths [1]. In indoor, RF signal is affected by multipath propagation. As a result shadowing and fading are occured which degrade the positioning accuracy. At presence of user where APs are placed at about $7 \mathrm{~m}$ apart, can change the standard deviation of received signal strength from $0.68 \mathrm{dBm}$ (without presence of user) to $3 \mathrm{dBm}[4]$.

\section{Positioning Technique}

RSS (received signal strength) fingerprint based positioning is prominent for $\mathrm{Wi}-\mathrm{Fi}$ based indoor positioning systems. In this approach the first phase is called off-line phase where received signal is captured at different locations and stored in a database against the position. The total area is divided into small boxes which are called grids where the measurement is taken for every grid. Once the total area is inspected for RSS fingerprinting, then the off-line phase is completed. Now the second phase is called: on-line phase, where the current captured RSS value is used to match with the stored RSS value (placed in a database) and for the position that it gets best match is considered as a current position.

\section{Matching Algorithm}

RSS is matched with suitable neighbour in the database in a manner that is governed by least Euclidean distance between current RSS and grid fingerprints during the online phase. For an example during off-line phase the RSS fingerprint is stored in a database like in Table I. 


\begin{tabular}{|l|l|l|l|l|}
\hline Grid Number & AP1 & AP2 & AP3 & AP4 \\
\hline Grid 1 & 60 & 65 & 65 & 50 \\
\hline Grid 2 & 80 & 85 & 85 & 60 \\
\hline$\ldots \ldots$ & $\ldots .$. & $\ldots .$. & $\ldots \ldots$ & $\ldots .$. \\
\hline Grid 256 & 55 & 45 & 65 & 80 \\
\hline
\end{tabular}

Table I: Sample data for RSS fingerprint.

Let's say, current captured RSS fingerprint is: $C R S S=$ $\{A P 1, A P 2, A P 3, A P 4\}=\{80,82,80,82,59\}$ during online phase. Now squared Euclidean distance is taken in account to find least euclidean distance using Equation 1.

$$
D_{\text {min }}=\sqrt{\sum_{i=1}^{4}\left[C R S S_{i}-R S S_{i}\right]^{2}}
$$

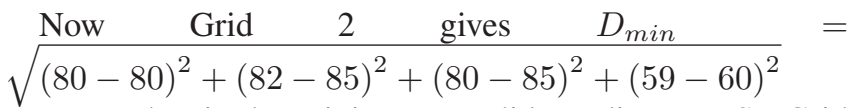
$=5.9$. That is the minimum Euclidean distance. So Grid 2 is the current location of the user.

\section{LIMITATIONS}

The mentioned straight forward technique can not bring error free positioning. This leads the unusual leapfrog of Wi-Fi RSS which miss-guides to find the correct location. This is because, Wi-Fi signal is very much susceptible to multipath fading in indoor. Moreover in indoor there are very large number of obstacles are placed randomly where there is a very high probability of Wi-Fi RSS fluctuation. To overcome this leapfrog nature of Wi-Fi, a probabilistic approach is necessary so that current location can be detected based on measured value and prediction. Filtering can provide a better accuracy and thus we are interested to apply the Particle Filter for positioning.

\section{Why PARTicle Filters?}

Signal level fluctuation is a natural behavior of Wi-Fi. Wi-Fi connectivity has unpredictable propagation in indoor environments. Most wireless positioning systems suffer from non-linear and non-Gaussian noise due to shadowing and existence of non-line-of-site propagation. Simple RSS based fingerprinting technique for Wi-Fi based indoor positioning can not perform well because of signal leap-frog phenomenon in indoor environments. As a result, it performs incorrect positioning and performs discontinuous trajectory of the movement of an asset within very small amount of time. Average fingerprint of received signal strength from different APs can bring a little improvement of accuracy but it cannot contribute to handle the fluctuation of received signal strength during the on-line phase (positioning phase). The use of Kalman Filter can lead to improve signal leapfrog phenomenon in indoor environments. However it cannot handle to halt to cross the obstacle (invalid trajectory of movement) during on-line phase, because it steps forward based on prediction. Somehow to improve the unrealistic trajectory crossing, a map is needed where the map contains information of the obstacles. Nevertheless this is very hard to implement for Kalman Filter [1]. Now Particle filter is a very adaptive filter which can overcome this problem easily. Besides, particle filter can easily handle non-linearity and non-Gaussian noise [6]. That's why we are interested on Particle Filters for indoor positioning purpose.

\section{PARTICLE FILTER}

The concept of Particle Filter is not very new. Previously, it was mostly used in visual tracking. Because of its attractive features, it is needed for Wi-Fi based indoor positioning systems where other traditional filters cannot fulfill the requirement. When we want to track the trajectory of an asset (human or robot), the measurement(observation) (see Figure 1) is not sufficient enough since it can contain errors.

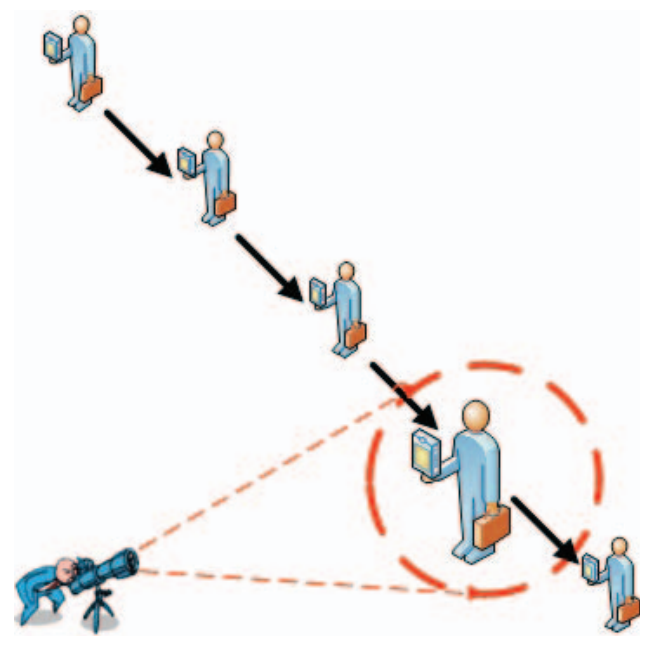

Figure 1: Observation(Measurement).

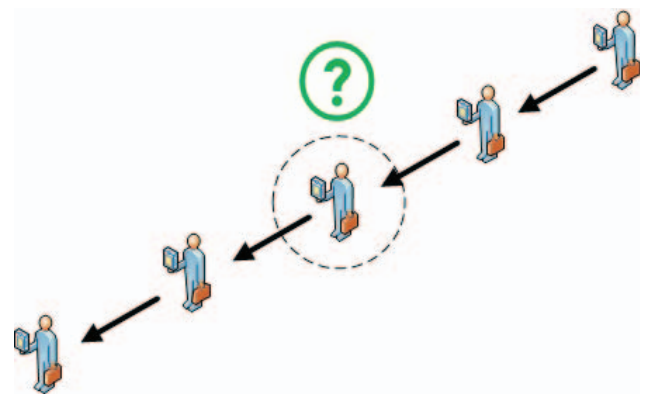

Figure 2: Prediction.

Again if we only rely on prediction of an asset based on previous history (see Figure 2), it is not practical because there is a big chance of miss-prediction of the asset. Moreover the prediction may be obsolete. If neither absolute prediction nor observation exists then what we can do? In 
this case combination of both observation and prediction can lead towards a better result (see the Figure 3) and Particle Filter also does the same thing.

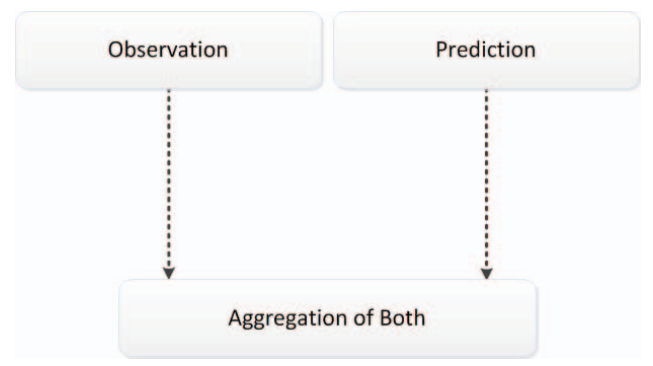

Figure 3: Particle filter concept.

Basically Particle Filter is a probabilistic tracking solution. It can be deliberated as an approximation to Bayesian recursive filtering [6]. In Particle Filter the posterior probability distribution of the current user is calculated and propagated using the set of weight samples [7]. Particle Filter composed of a good number of particles and collection of particles make a particle cloud. Particles are the representative of the traced position. Each particle has its own weight. This weight is responsible to consider a particle to take part into positioning.

\section{Particles state illustration}

Every time Gaussian noise is added to the position of a particle continuously. Particles are formed based on Monte Carlo simulations where the main idea is to generate random samples from Gaussian probability distribution. Two uniformly distributed $\mathcal{U}[0,1]$ random numbers $x_{1}$ and $x_{2}$ are generated. These two numbers are passed through the BoxMuller transformation equation so that we can find samples from Gaussian distributions (see the equations below).

$$
\begin{aligned}
& x=\sqrt{-2 \ln \left(x_{1}\right)} \cos \left(2 \pi x_{2}\right) \\
& y=\sqrt{-2 \ln \left(x_{1}\right)} \sin \left(2 \pi x_{2}\right)
\end{aligned}
$$

A particle is composed of different elements where those elements represent state $\left(s_{t}\right)$. A particle can change its state by changing its weight, position and orientation. A particle at any time $\mathrm{t}$ can have maximum weight, $\mathrm{W}=1$; orientation angle, $\theta=\{n \mid n \in(0,2 \pi)\}$ and position, $P=$ $\{x, y \mid x \in$ mapWidth, $y \in$ mapHeight $\}$.

Like HMM, hidden states and observation states are available in Particle Filter where hidden state is represented by particle's state and observation state is represented by observed received signal strength for positioning as shown in figure 5 where a state at time $\mathrm{k}$ is denoted by $s_{k}$ and observation state is denoted by $o_{k}$.

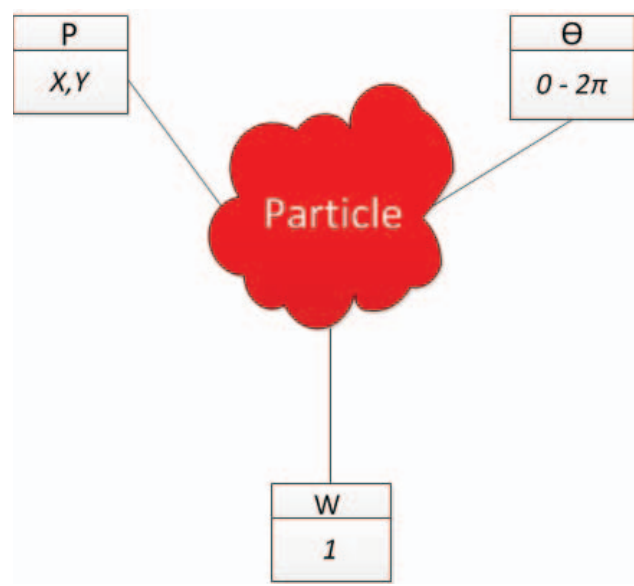

Figure 4: Particle state illustration.

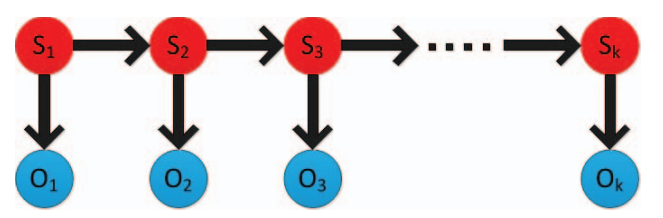

Figure 5: HMM approach in PF.

\section{Motion MOdEL: PARTICLES MOTION ILLUSTRATION}

Particles follow the motion model to change their state when it is needed. In motion model, particles can change their state by changing positions and orientations. A particle's current orientation, next orientation and changes in orientation are denoted by $\theta_{t}, \theta_{t+1}, \delta \theta_{t+1}$ respectively. Probability of next orientation of a particle when current orientation is true can be denoted by $P\left(\theta_{t+1} \mid \theta_{t}\right)$ and can be determined based on $\delta \theta_{t+1} . \delta \theta_{t+1}$ can be earned by sampling from Gaussian probability distribution $N\left(0, \sigma_{\theta}^{2}\right)$ where $\sigma_{\theta}^{2}$ represents variance of motion errors [7]. Now a particle's current position, next position and change in position are denoted by $P_{t}, P_{t+1}, \delta P_{t+1}$ respectively, where $P_{t}=\left\{x_{t}, y_{t}\right\}, P_{t+1}=\left\{x_{t+1}, y_{t+1}\right\}$.

$$
\begin{aligned}
& x_{t+1}=x_{t}+\delta x_{t+1}=x_{t}+\delta D * \cos \left(\theta_{t}\right) \\
& y_{t+1}=y_{t}+\delta y_{t+1}=y_{t}+\delta D * \sin \left(\theta_{t}\right)
\end{aligned}
$$

Here $\delta D$ represents the displacement of a particle (see, Figure 6) in motion model can be obtained by sampling from a Gaussian Distribution $N\left(\mu_{D}, \sigma_{D}^{2}\right)$ [7].

\section{ObSERVATion Model: PARticles update ILLUSTRATION}

Now let's say we have a set of collected observations from time 1 to $\mathrm{t}$ that can be denoted as $o_{1: t}$. Now prior 


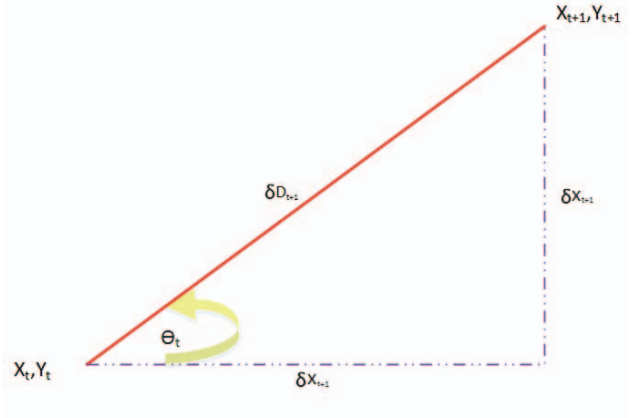

Figure 6: Particle movement.

pdf $\operatorname{Pr}\left[s_{t-1} \mid o_{1: t-1}\right]$, the posterior pdf $\operatorname{Pr}\left[s_{t} \mid o_{1: t}\right]$ can be formulated based on Bayes rule [8]:

$$
\operatorname{Pr}\left[s_{t} \mid o_{1: t}\right]=\frac{\operatorname{Pr}\left[o_{t} \mid s_{t}\right] \operatorname{Pr}\left[s_{t} \mid o_{1: t-1}\right]}{\operatorname{Pr}\left[o_{t} \mid o_{1: t-1}\right]}
$$

But in practical it is imposible to calculate exact posterior pdf $\operatorname{Pr}\left[s_{t} \mid o_{1: t}\right]$, but it can be approximated. When there are large number of particles then pdf can be incorporated to [5]:

$$
\operatorname{Pr}\left[s_{t} \mid o_{1: t}\right]=\sum_{n=1}^{N} w_{t}^{n} \delta\left(s_{t}-s_{t}^{n}\right)
$$

Now received signal strength plays a vital role for WiFi based indoor positioning system. Observation Model is followed by Particle Filter. Observation model describes the probability of observation at different places when particles current state information is available and at that time Wi-Fi received signal strength is also taken into account to find out any necessary correction of states. Probability of observation or measurement at time $t$ when current state of a particle is known can be denoted by $\operatorname{Pr}\left[o_{t} \mid s_{t}\right]$ and can be defined as follows:

$$
\operatorname{Pr}\left[o_{t} \mid s_{t}\right]=\frac{1}{\sqrt{2 \pi \sigma}} \exp \left[-\frac{\left(P_{o t}-P_{s n}\right)^{2}}{2 \sigma^{2}}\right]
$$

Where $P_{o t}$ represents the position after observation that can be returned from fingerprint database when least Euclidean distance for RSS is calculated, $P_{s n}$ represents the position of the nth particle and $\sigma$ denotes variance of measurement. Actually small value of $\sigma$ indicates small variations of positions for different particles at the same position and small $\sigma$ leads to small correction in movement for different particles.

\section{Working STEPS OF PARTICLE FILTER FOR POSITIONING}

Particle Filter follows the following steps:

W Step 1: Particles are generated by sampling from the Gaussian probability distribution and finally formed particle cloud. Average position of particle cloud points the actual position. The weights of all particles are normalized so that sum of all particles weight is equal to 1 .

Step 2: All particles are followed by the motion model where states of particles are predicted.

Step 3: All particles are followed by the observation model where particles movement are corrected. Then the weights of particles are normalized to 1 .

Step 4: If weight of any particle is below the threshold then it needs a re-sample again like Step 1.

* Step 5: Go to Step 2 and continue the motion model and follow afterwards .

\section{Performance EVAluation of the PROTOTYPE}

We developed a user friendly prototype for Wi-Fi based indoor positioning. The prototype can easily be run from a Windows PC or a Laptop with .NET environment. Using this prototype a user can pinpoint its location in indoor.

\section{EXPERIMENTAL AREA}

We performed experiments on passage of ground floor of Tieto Sweden AB in Luleå, Sweden. The area of test-bed is near around $400 \mathrm{~m}^{2}$ (see Figure 7). There are 4 access points are placed on the testbed where all of those access points can be easily sensed by WiFi users. We used NETGEAR N150 Wireless Router as an access point. It supports IEEE 802.11 $\mathrm{b} / \mathrm{g} / \mathrm{h}$ standards and works on $2.4 \mathrm{GHz}$ frequency band. Apart from 4 APs, some APs are detected through scan where some of those are placed in different floors in this building and some are from neighbouring buildings. But these APs are not considered for positioning because they are unstable. So during the off-line phase, 4 APs are selected to construct calibration data. During on-line phase the positioning is performed based on 4 APs. The measurements are taken at non working hours where in absence of people makes it as a static environment. Now for this testbed the user having a device (PC) with wireless card is performed measurement as well as positioning work. In total 51 calibration points are used to represent calibrated data and calibration points are placed at about $1 \mathrm{~m}$ spaced to each neighbouring point. Each calibration point is taken in 4 directions like up, down, left and right directions and as well as taken in only one direction separately.

And during off-line phase for each point the scan is continued until the 4 APs are available at the same time and after that for that point RSS is averaged for each AP. The maximum recorded RSS is $0 \mathrm{dbm}$ and minimum is $-89 \mathrm{dbm}$ and after averaging it's maximum is $-27 \mathrm{dbm}$ and minimum is $-82.8 \mathrm{dbm}$. Now during the online phase which means 


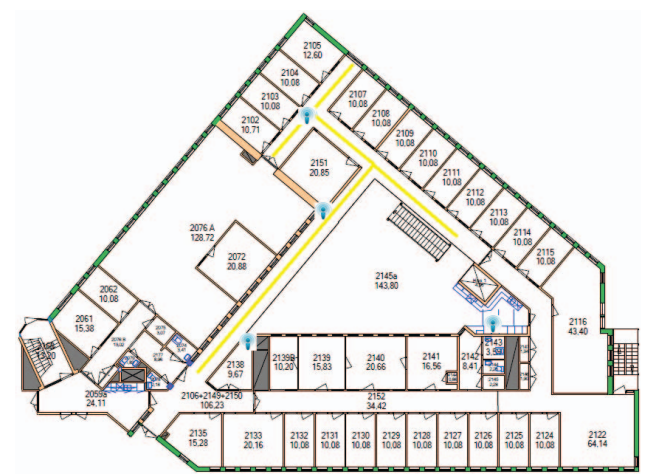

Figure 7: Testbed environment.

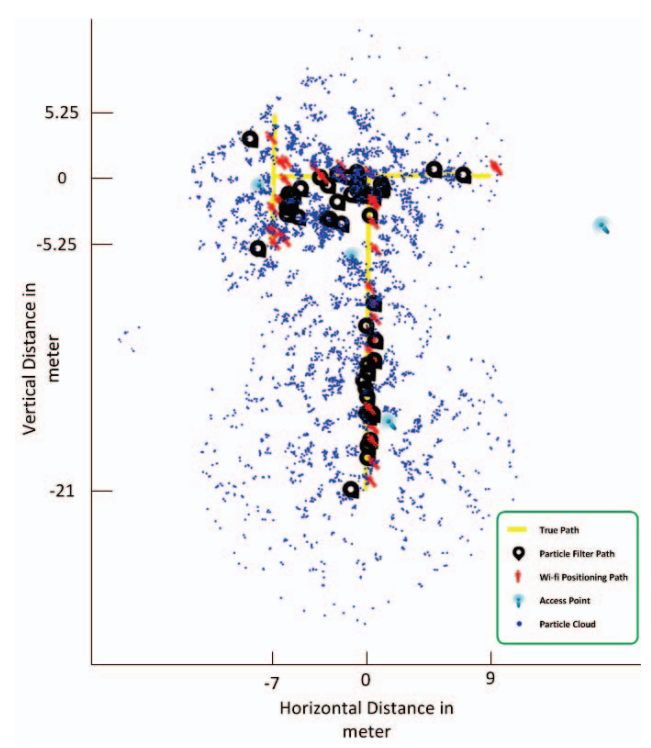

Figure 8: Paticles generation on the screen.

positioning phase where the user moved in straight direction from starting point to end point (yellow coloured line in Figure 8) with a normal walking velocity. So yellow colored line represents true path of movement, the red man icon says the current position of user using Wi-Fi RSS fingerprint method and black pin indicates positioning when we applied Particle Filter and the blue dots represent particles where total number of particles are formed a particle cloud. Particle Filter performed positioning which is the mean position of particles in a cloud. The prototype updates user location in every 2 seconds. It has been observed that calibration data in 4 directions has a better result comparing with calibration data in one direction because little change in orientation of Wi-Fi user can result RSS fluctuation (see Table II). On the other hand, constructing calibration data in 4 directions requires more times comparing with one direction.

Now if we reduce one AP from available APs that means in total 3 APs and perform positioning for that then it performs worse comparing with 4 APs (see figure 9 and
$10)$ as 3 APs result a mean distance error of $4.32 \mathrm{~m}$ where 4 APs result a mean distance error of $2.41 \mathrm{~m}$ (see Table II). Now RSS fingerprint method without Particle Filter doesn't perform well, since it fluctuates abruptly which results error in distance.

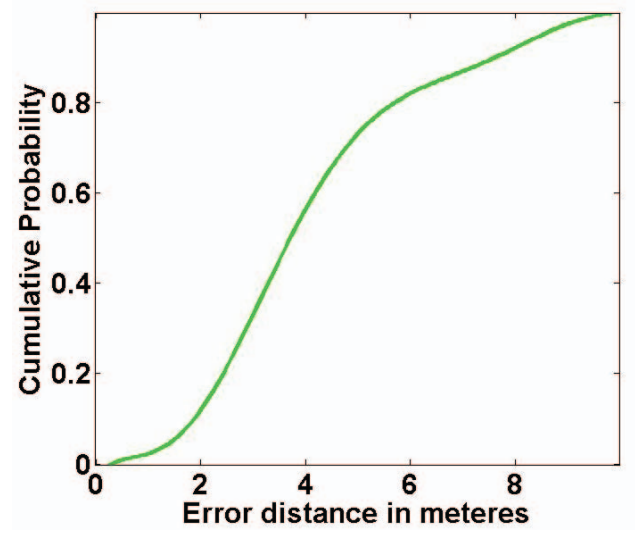

Figure 9: Cumulative error in distance for 3 APs.

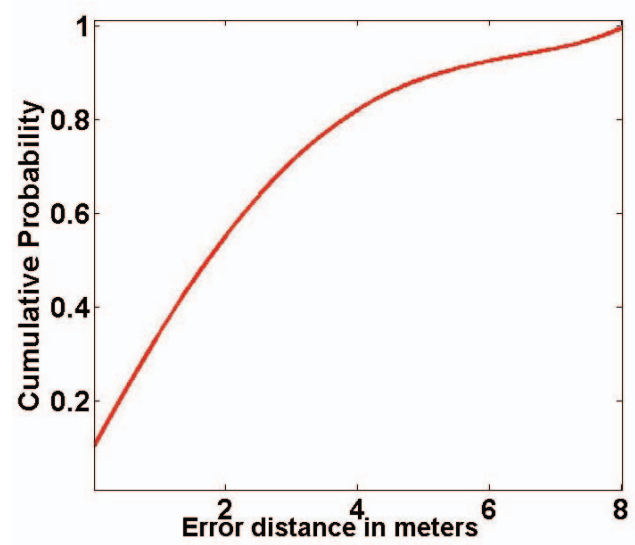

Figure 10: Cumulative error in distance for 4 APs.

\begin{tabular}{|l|l|l|l|}
\hline & Mean & Median & St.deviation \\
\hline 4 AP, 4 directions calibration data (only WiFi RSS) & 3.6 & 2.25 & 3.17 \\
\hline 4 AP, 4 directions calibration data (with PF) & 2.41 & 2 & 2.22 \\
\hline 4 AP, one direction calibration data (with PF) & 4.59 & 3 & 4.23 \\
\hline 3 AP, 4 directions calibration data (with PF) & 4.32 & 4 & 2.17 \\
\hline
\end{tabular}

Table II: Distribution information of distance error in meters for different cases.

Again positioning accuracy also depends on number of particles (N) generation. Here we found for $\mathrm{N}=400,600$ particles gives better accuracy comparing to $\mathrm{N}=100,300$, 800 and N=600 is the best (see Figure 11,12 and Table III). 


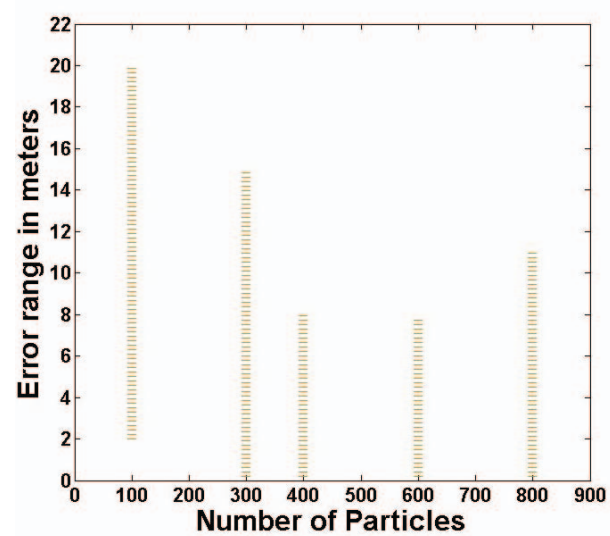

Figure 11: Error ranges for different number of particles.

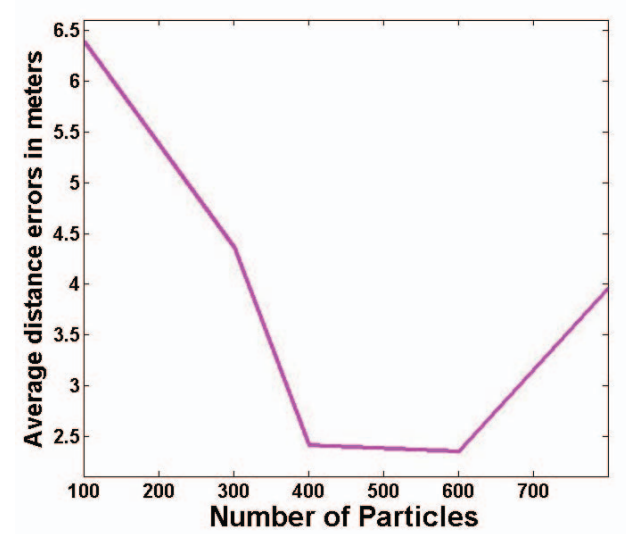

Figure 12: Mean error distance in meters for different number of particles.

\begin{tabular}{|l|l|l|l|l|l|}
\hline & Min & Max & Mean & Median & Standard deviation \\
\hline PF, $\mathrm{N}=100$ & 2 & 20 & 6.39 & 6 & 3.32 \\
\hline PF, $\mathrm{N}=300$ & 0 & 15 & 4.36 & 4 & 3.09 \\
\hline PF, $\mathrm{N}=400$ & 0 & 8 & 2.41 & 2 & 2.22 \\
\hline PF, $\mathrm{N}=600$ & 0 & 8 & 2.35 & 2 & 2.07 \\
\hline PF, $\mathrm{N}=800$ & 0 & 11 & 3.96 & 3.37 & 3.13 \\
\hline
\end{tabular}

Table III: Distribution information of distance error in meters for varying number of particles in Particle Filter.

\section{CONClusion}

The necessity of PF (Particle Filter) is properly investigated through experiment that we described above. Based on experiment we can conclude that PF can bring noticeable improvement relative to stand-alone Wi-Fi based indoor positioning method during the positioning time. The selection of number of particles is also an important matter. Best performance is achieved at $\mathrm{N}=600$ although it needs computational delay. On the other hand generation of calibration data is also a vital factor, during off-line phase. Our experiment suggested that in order to get a good accuracy, calibration data should be constructed into four directions.
Our future study will be to conduct experiment and observe the error, after the aggregation of digital compass with this PF. In addition we are also interested to perform investigation of Voroni Filter to find how it can contribute to minimize the error for our prototype.

\section{REFERENCES}

[1] W.H.Wong, J.K.Ng and W.M Yeung, "Wireless LAN positioning with mobile devices in a library environment", 25th IEEE International Conference on Distributed Computing Systems Workshops, 2005. pp. 633- 636, 6-10 June 2005.

[2] http://geta.aalto.fi/en/courses/bringing_navigation_indoors.pdf

[3] K.Ogunjemilua, J.N.Davies, V.Grout and R.Picking, "An Investigation into Signal Strength of 802.11n WLAN", Proceedings of the Fifth Collaborative Research Symposium on Security, E-Learning, Internet and Networking (SEIN 2009), Darmstadt, Germany, 26-27 November 2009, pp. 191-204.

[4] M.N.A.Ngan, M.A.Karim, B.Parhizkar, A.H.Lashkari, "Mobile WiFi-Based Indoor Positioning System", International Journal of Computer Science and Information Security, vol. 10, No. 3,13-22 March 2012.

[5] F.Evennou, F.Marx, and E.Novakov, "Map-aided indoor mobile positioning system using particle filter", IEEE Wireless Communications and Networking Conference, New Orleans, LA USA, 13-17 March 2005.

[6] Z.Shah, R.A.Malaney, X.Wei and K.Tai , "Experimental Deployment of Particle Filters in WiFi Networks", IEEE International Conference on Communications- ICC 2007, pp. 46924697, 24-28 June 2007.

[7] I.Kim, E.Choi and H.Oh, "Observation and motion models for indoor pedestrian tracking", 2012 Second International Conference on Digital Information and Communication Technology and it's Applications (DICTAP), pp. 482-485, 16-18 May 2012.

[8] C.Florin, N.Paragios, J.Williams, "Monte-carlo Sampling, Particle Filters and Segmentation of Coronaries", Research Report 05-03, January 2005. 